\title{
Rapid Phase Locking in Systems of Pulse-Coupled Oscillators with Delays
}

\author{
Wulfram Gerstner \\ Department of Zoology, University of Oxford, Oxford OX1 3PS, England \\ and Physik Department der TU München, D-85747 Garching bei München, Germany*
}

(Received 3 August 1995)

\begin{abstract}
The dynamical evolution of a system of integrate-and-fire units with delayed excitatory coupling is analyzed. The connectivity is arbitrary except for a normalization of the total input to each unit. It is shown that the system converges to a periodic solution where all units are phase locked but do not necessarily fire in unison. In the case of discrete and uniform delays, a periodic solution is reached after a finite time. For a delay distribution with finite support, an attractor is, in general, only reached asymptotically.
\end{abstract}

PACS numbers: 87.10.+e, 05.20.-y, 64.60.Ht

Networks of pulse-coupled oscillators have attracted an increasing amount of interest [1-6]. Theoretical results on synchronization and phase locking have been applied to a wide range of phenomena including synchronously flashing fireflies [7], biological clocks [8], oscillating neuronal activity [9], and earthquake cycles [10]. Most of the analytical studies [1,2] have focused on fully connected networks where mean-field methods can be applied. In a different line of research aiming at an understanding of self-organized criticality, networks with local connections have been studied [4-6]. Recently, it has been shown that homogeneous networks of integrate-and-fire units with arbitrary, local or long-ranged, connectivity and no leakage are amenable to mathematical analysis whenever the total input to each unit is normalized [3]. In the model network presented below we use this general class of connectivity.

In systems of identical integrate-and-fire units without leakage, many degenerate cyclic solutions with the same period can coexist [3-5]. The convergence time to the set of periodic solutions is short. More precisely, a periodic solution is reached as soon as every unit has fired once [3]. This result, however, is limited to networks with delayless interaction and instantaneous reset of the state variable after each firing. Naturally, the question arises whether fast phase locking is specific for the delayless situation or generic in the sense that it holds for a broader class of oscillator models.

In this paper, oscillator networks with delayed excitatory interaction and partially delayed reset are studied. Such systems can be considered as extremely simplified models of neural networks or earthquake faults [3]. There are several questions concerning the dynamics of such systems. Are there periodic solutions? If so, what is their period? What is the asymptotic system behavior? How fast is an attractor reached? These questions are addressed below. It is shown that all attractors are periodic. As in the delayless case, units are phase locked but not necessarily in synchrony. Furthermore, the attractors are reached after a finite time, if delays are discrete and shorter than the period of a cyclic solution.
We consider a network of $N$ integrate-and-fire units $1 \leq$ $i \leq N$. Each unit is described by a scalar variable $u_{i}$ which increases, in the case of no interaction, at a constant rate. Without loss of generality, we set $d u_{i} / d t=1$. To be specific, we can think of $u_{i}$ as the membrane potential of a neuron without leakage driven by a constant input current $I_{0}=1$. In the alternative interpretation of a stick-slip model of an earthquake fault, $u_{i}$ is the accumulated stress or friction force of unit $i$ in a network of interconnected blocks which are pulled over a rough surface. If $u_{i}$ reaches a threshold $\vartheta=1$, a pulse is generated. This defines a firing time $t_{i}^{f}$. In a neuronal interpretation, firing refers to the emission of an action potential; in an earthquake model, firing corresponds to the sliding of a single block. Firing has two effects. First, it causes a sharp drop of the state variable $u_{i}$ due to an internal reconfiguration pulse $-\gamma_{i}\left(t-t_{i}^{f}\right)$. Second, other units $k$ receive, after some delay, a positive signal pulse $J_{k i} \alpha_{k i}\left(t-t_{i}^{f}\right)$. The factor $J_{k i}$ is a measure of the effectivity of signal transmission from $i$ to $k$. To account for causality, we have $\alpha_{i j}(s)=$ $\gamma_{i}(s)=0$ for $s \leq 0$.

The full dynamics of a unit $i$ in a network of $N$ oscillators is given by

$\frac{d}{d t} u_{i}(t)=1-\sum_{f} \gamma_{i}\left(t-t_{i}^{f}\right)+\sum_{j} \sum_{f} J_{i j} \alpha_{i j}\left(t-t_{j}^{f}\right)$,

where $t_{i}^{f}$ denotes the times where $u_{i}$ reaches 1 from below. The sum runs over all firing times $t_{i}^{f}$ or $t_{j}^{f}$ with $t_{i}^{f}, t_{j}^{f}<t$ and, respectively, all units $1 \leq j \leq N$. Without loss of generality we assume a normalization $\int_{0}^{\infty} \alpha_{i j}(s) d s=\int_{0}^{\infty} \gamma_{i}(s) d s=1$ for all $1 \leq i, j \leq N$.

Equation (1) defines the dynamics of a network of integrate-and-fire units under quite general conditions. In the following, we impose a number of requirements concerning the coupling parameters $J_{i j}$ and the function $\gamma_{i}(s)$ and $\alpha_{i j}(s)$. First, as in [3], we require that none of the couplings $J_{i j}$ is negative $\left(J_{i j} \geq 0\right)$ and that the total input to every unit is normalized

$$
\sum_{j} J_{i j}=A<1 \quad \text { for all } i \text { with } 1 \leq i \leq N .
$$


Second, the functions $\alpha_{i j}$ and $\gamma_{i}$ should be pulselike and positive. Specifically, we require for $\alpha_{i j}(s)$

$$
\alpha_{i j}(s) \geq 0 ; \quad \alpha_{i j}(s)=0 \quad \text { for } s \geq \Delta
$$

with a maximum delay $\Delta<1-A$. Similarly, we require

$$
\gamma_{i}(s) \geq 0 ; \quad \gamma_{i}(s)=0 \quad \text { for } s \geq \Delta,
$$

where $\gamma_{i}$ is of the form $\gamma_{i}(s)=\gamma_{i}^{0} \delta(s)+\gamma_{i}^{1}(s)$ with $\gamma_{i}^{0}>A$ and $\gamma_{i}^{1}(s)$ decreasing; $\delta(\cdot)$ denotes the Dirac $\delta$ function. The term $\gamma_{i}^{0} \delta(s)$ ensures that the firing times of each unit are separated by finite intervals. In a neuronal interpretation, $\gamma_{i}^{0}$ can be related to absolute refractoriness. Similarly, the contribution $\gamma_{i}^{1}(s)$ describes relative refractoriness. In the stick-slip model of interconnected blocks, $\gamma_{i}^{0}$ corresponds to instantaneous and $\gamma_{i}^{1}$ to slow stress release. Since conditions (2)-(4) are fairly abstract, we present two illustrations.

(I) As a first example, let us consider a fully connected network of integrate-and-fire units (1) with $J_{i i}=0$ and homogeneous coupling $J_{i j}=J_{0} /(N-1)$ for $j \neq i$ with $0<J_{0}<1$. If $u_{i}(t)$ reaches the threshold $\vartheta=1$ at $t=t_{i}^{f}$, the state variable is reset to $\lim _{\delta \rightarrow 0} u_{i}\left(t_{i}^{f}+\delta\right)=$ 0 . The reset is equivalent to a reconfiguration pulse $\gamma_{i}(s)=\delta(s)$, that is, $\gamma_{i}^{0}=1$ and $\gamma_{i}^{1}=0$. Due to the firing event at $t_{i}^{f}$, all other units receive an identical signal pulse $\alpha_{j i}(s)=\alpha(s)$. To be specific, we assume that the signal is a square pulse of duration $\Delta_{0} \leq 1-J_{0}$ which arrives with zero delay. Thus,

$$
\alpha(s)=\Delta_{0}^{-1} \theta(s) \theta\left(\Delta_{0}-s\right),
$$

where $\theta(s)$ denotes the Heaviside step function $\theta(s)=0$ for $s \leq 0$ and $\theta(s)=1$ for $s>0$. Such a network meets the requirements (2)-(4).

(II) As a second, and more interesting, example, let us consider a two-dimensional lattice of integrate-andfire units with finite-range couplings. As before we have $\gamma_{i}(s)=\delta(s)$, but there are now two different types of signals $J_{i j} \alpha_{i j}(s)$. First, there is strong input from a group $G_{i}^{(1)}$ of $n^{(1)}$ units around unit $i$. The pulses from neighbors $j \in G_{i}^{(1)}$ arrive with a delay $\Delta^{(1)}$ and have a strength $J_{i j}=$ $J^{(1)}>0$. A second group $G_{i}^{(2)}$ farther apart contains $n^{(2)}$ neighbors $j$ with weaker coupling $J^{(2)}$ with $0<J^{(2)}<$ $J^{(1)}$ and longer delays $\Delta^{(2)}>\Delta^{(1)}$. Assuming negligible pulse width, we have

$$
\alpha_{i j}(s)=\delta\left(s-\Delta^{(m)}\right) \text { for } j \in G_{i}^{(m)},
$$

where $m=1,2$. As long as the numbers $n^{(1)}$ and $n^{(2)}$ are identical for all units $1 \leq i \leq N$, condition (2) holds with $A=n^{(1)} J^{(1)}+n^{(2)} J^{(2)}$. We require $A<1$ and $\Delta^{(2)}<$ $1-A$. The network also meets the other requirements. The above examples illustrate that conditions (2)-(4) hold for a large class of model systems including locally and globally coupled networks.

We are interested in the global dynamics of a system of coupled oscillators defined by (1)-(4). The system is started at $t=0$ with a set of initial values $0 \leq u_{i}(0)<1$.
We assume that there have been no firing events in an interval of length $\Delta$ preceding $t=0$. We would like to understand the asymptotic network behavior. How fast is an attractor reached? If there are periodic limit cycles, what is the period? The above questions are answered by the theorem stated below.

We focus on the interval $P_{k}^{f}=t_{k}^{f+1}-t_{k}^{f}$ between two consecutive pulses of a unit $k$. Since all couplings $J_{k j}$ are positive, the interval $P_{k}^{f}$ is always shorter than the interval $P_{0}=1$ of the free oscillator. In particular, in a time window of length $2 P_{0}$, each oscillator emits at least two pulses. In the following, it will be shown that the longest interval that can be found in a search window $\left[t-2 P_{0}, t\right]$ is always decreasing. More precisely, we define $P_{\max }(t)=P_{0}$ for $t<2 P_{0}$ and

$$
P_{\max }(t)=\max _{k, f}\left\{t_{k}^{f+1}-t_{k}^{f} \mid t-2 P_{0} \leq t_{k}^{f}, t_{k}^{f+1} \leq t\right\}
$$

for $t \geq 2 P_{0}$.

Theorem.-Assume that a network defined by (1)(4) is started at $t=0$ with the initial values $u_{i}(0)$ and that there are no firings in the time window $[-\Delta, 0]$. Then the following holds: (i) $P_{\max }(t)$ as defined in (7) is nonincreasing. (ii) $\lim _{t \rightarrow \infty} P_{\max }(t)=1-A$. This is the minimum interval between consecutive pulses. (iii) If $P_{\max }(t)$ stays constant during a time $t_{0} \leq t<t_{0}+\Delta$ with $t_{0} \geq 2 P_{0}$, then a periodic solution has been reached and $P_{\max }\left(t_{0}\right)=1-A$.

An application of the theorem to the above examples yields the following

Corollary.-In the network of example (II), a periodic solution with period $1-A$ is reached in a time shorter than $2 P_{0}+\left(n^{(1)}+1\right)\left(n^{(2)}+1\right) \Delta$. In a network as in example (I), a periodic solution is, however, in general only reached asymptotically.

Since $P_{\max }(t)$ is non-negative, statement (i) implies that $P_{\max }(t)$ is a Lyapunov function of the network dynamics. From (ii) we conclude that on an attractor, all units fire with the same period $1-A$. Note that there are many different periodic solutions consistent with this condition, i.e., units can fire synchronously or in some fixed order depending upon the initial conditions. Statement (iii) is a simple criterion to decide whether an attractor has been reached. It can be used to derive the upper bound for the convergence time to an attractor which is stated in the first part of the corollary. A generalization to other connection topologies with more than two groups of neighbors is straightforward. We note that with zero delay, a periodic solution is always reached before the time $2 P_{0}$. In fact, for delayless interaction, an attractor is reached as soon as every unit has fired once [3].

We emphasize that, for pulses $\alpha_{i j}(s)$ of finite width, a periodic solution is, in general, only reached asymptotically. To illustrate this point, let us consider a simple network of two units with pulses $\alpha(s)$ and $\gamma(s)$ as in example (I). The interaction is given by (5) with $\Delta_{0}=1-A$ 
and strength $J_{12}=J_{21}=A$ and $J_{11}=J_{22}=0$. The initial conditions are $u_{1}(0)=u_{0}$ with $0<u_{0}<1$ and $u_{2}(0)=$ 0 . A straightforward calculation shows that the first few pulses occur at $t_{1}^{1}=1-u_{0}, t_{2}^{1}=1-A u_{0}$ and $t_{1}^{2}=$ $t_{1}^{1}+(1-A)\left(1+A u_{0}\right), t_{2}^{2}=t_{2}^{1}+(1-A)\left(1+A^{2} u_{0}\right)$. The following intervals $P_{k}^{f^{2}}=t_{k}^{f^{2}+1}-t_{k}^{f}$ are given by the equation

$$
P_{k}^{f+1}-P_{k}^{f}=-\left(1-A^{2}\right)\left[P_{k}^{f}-(1-A)\right],
$$

where $k=1,2$ and $f \geq 1$. Thus a periodic solution with $P_{k}^{f} \equiv 1-A$ is only reached asymptotically. Moreover, the typical number $\bar{f}$ of firings per unit which are needed to reduce the distance $x=P_{k}^{f}-(1-A)$ from the attractor by a factor of $1 / e$ diverges as $A \longrightarrow 1$. Nevertheless, the time scale of (asymptotic) convergence to the periodic solution, defined as $\bar{t}=\bar{f}(1-A) \propto(1+A)^{-1}$ remains bounded and is of order 1 , whatever $A$. In this sense, there can be rapid phase locking even for networks with interaction pulses of finite width.

We stress that the theorem applies to networks with excitatory coupling only. For inhibitory connections or mixtures of excitation and inhibition, the situation can be quite different. As a simple example, we consider a network with inhibitory coupling of strength $\sum_{j} J_{i j}=$ $A<0$ and $|A|<1$, where all connections have the same long delay $\alpha_{i j}(s)=\delta(s-\Delta)$ with $1<\Delta<1-A ; \delta(\cdot)$ denotes the Dirac $\delta$ function, and we assume instantaneous reset $\gamma_{i}(s)=\delta(s)$. If all units are started with identical initial conditions, they remain synchronous thereafter. A straightforward consideration shows that the interspike intervals do not approach a constant value but alternate between 1 and $1-2 A$. Thus, in contrast to the excitatory case, the final state has the minimal period $2(1-A)$, and $P_{\max }(t)$ as defined in (7) oscillates. The oscillation of interspike intervals is due to a generic instability of inhibitory systems: A unit which has received a first inhibitory input needs more time to reach the threshold and is therefore prone to receiving even more inhibition.

After these remarks, let us now turn to the proof of the theorem. It proceeds in five steps and takes the rest of the paper.

Step 1. - Integration of (1) yields

$$
\begin{aligned}
u_{i}(t)= & t+u_{i}(0)-\sum_{f} \eta_{i}\left(t-t_{i}^{f}\right) \\
& +\sum_{j} J_{i j} \sum_{f} \epsilon_{i j}\left(t-t_{j}^{f}\right),
\end{aligned}
$$

with $\eta_{i}(s)=\epsilon_{i j}(s)=0$ for $s \leq 0$ and $\eta_{i}(s)=$ $\int_{0}^{s} \gamma_{i}\left(s^{\prime}\right) d s^{\prime}, \quad \epsilon_{i j}(s)=\int_{0}^{s} \alpha_{i j}\left(s^{\prime}\right) d s^{\prime} \quad$ for $\quad s>0$. The summation over $f$ runs over all firing times with $0 \leq t_{i}^{f}<t$ and $0 \leq t_{j}^{f}<t$, respectively. Due to conditions (3) and (4), both $\eta_{i}$ and $\epsilon_{i j}$ are increasing functions. Furthermore, because of the normalization of $\alpha_{i j}$ and $\gamma_{i}$, we have $\eta_{i}(s)=\epsilon_{i j}(s)=1$ for $s \geq \Delta$.

Step 2.-We show that the minimum interval between two pulses is longer than or equal to $1-A$. To do so, let us consider the shortest interval that has occurred before time $t, P_{\min }(t)=\min _{k, f}\left\{t_{k}^{f+1}-t_{k}^{f} \mid 0 \leq t_{k}^{f}<\right.$ $\left.t_{k}^{f+1} \leq t\right\}$. We choose a unit $i$ and a firing time $t_{i}^{F+1} \leq t$ such that $t_{i}^{F+1}-t_{i}^{F}=P_{\min }(t)$. Since firing requires $u_{i}\left(t_{i}^{f}\right)=1$ immediately before pulse emission, it follows that $u_{i}\left(t_{i}^{F+1}\right)-u_{i}\left(t_{i}^{F}\right)=0$. Using Eq. (9), we find

$$
0=P_{\min }(t)-\Delta \eta_{i}+\sum_{j} J_{i j} \Delta \epsilon_{i j},
$$

with

$$
\begin{aligned}
\Delta \eta_{i} & =\sum_{f}\left[\eta_{i}\left(t_{i}^{F+1}-t_{i}^{f}\right)-\eta_{i}\left(t_{i}^{F}-t_{i}^{f}\right)\right], \\
\Delta \epsilon_{i j} & =\sum_{f}\left[\epsilon_{i j}\left(t_{i}^{F+1}-t_{j}^{f}\right)-\epsilon_{i j}\left(t_{i}^{F}-t_{j}^{f}\right)\right] .
\end{aligned}
$$

Reordering of the terms yields

$$
\begin{aligned}
\Delta \eta_{i}= & \eta_{i}\left(t_{i}^{F+1}-t_{i}^{F}\right) \\
& +\sum_{f=1}^{F-1}\left[\eta_{i}\left(t_{i}^{F+1}-t_{i}^{f}\right)-\eta_{i}\left(t_{i}^{F}-t_{i}^{f}\right)\right] .
\end{aligned}
$$

Since $\eta_{i}(s)$ is increasing, we find $\Delta \eta_{i} \geq \eta_{i}\left(P_{\text {min }}\right)$. Similarly,

$$
\begin{aligned}
\Delta \epsilon_{i j}= & \epsilon_{i j}\left(t_{i}^{F+1}-t_{j}^{1}\right) \\
& +\sum_{f=1}\left[\epsilon_{i j}\left(t_{i}^{F+1}-t_{j}^{f+1}\right)-\epsilon_{i j}\left(t_{i}^{F}-t_{j}^{f}\right)\right],
\end{aligned}
$$

with $t_{i}^{F+1}=t_{i}^{F}+P_{\min }$ and $t_{j}^{f+1}>t_{j}^{f}+P_{\min }$. Because $\epsilon_{i j}(s)$ is increasing, the second term is nonpositive. The first term is bounded by one and thus $\Delta \epsilon_{i j} \leq 1$. Using these results in Eq. (10), we find

$$
P_{\min } \geq \eta_{i}\left(P_{\min }\right)-A .
$$

Since, due to condition (4), $\lim _{\delta \rightarrow 0} \eta_{i}(\delta)>A, \eta_{i}(\Delta)=$ 1 with $\Delta<1-A$, and $d^{2} \eta_{i} / d s^{2} \leq 0$, it follows that $P_{\text {min }} \geq 1-A$.

Step 3. - We show that if $P_{\max }(t)$ as defined in (7) increases at a time $t^{*}$, it either increases farther at a later time $t^{\prime}$ with $t^{*}<t^{\prime}<t^{*}+P_{0}$ or we have $P_{\max }\left(t^{*}\right) \leq$ $1-A$. Let us assume that $P_{\max }$ increases at a time $t^{*}$ and does not increase afterwards. This implies that there is a unit $i$ with $t_{i}^{F+1}=t^{*}$ and $t_{i}^{F+1}-t_{i}^{F}=P_{\max }\left(t^{*}\right)$ and $P_{\max }(t) \leq P_{\max }\left(t^{*}\right)$ for $t>t^{*}$. Since $u_{i}\left(t_{i}^{F+1}\right)-$ $u_{i}\left(t_{i}^{F}\right)=0$, we have with (11) and (12)

$$
0=P_{\max }\left(t^{*}\right)-\Delta \eta_{i}+\sum_{j} J_{i j} \Delta \epsilon_{i j} .
$$

We derive bounds for $\Delta \eta_{i}$ and $\Delta \epsilon_{i j}$. Because all arguments on the right-hand side of (11) are not smaller than $1-A$ and $\eta_{i}(1-A)=1$, Eq. (11) yields $\Delta \eta_{i}=1$. In order to find a lower bound for (12), we split the firing times $t_{j}^{f}$ of a given unit $j$ into two groups. The first group $\Gamma_{1}$ contains firings before $t_{i}^{F}-\Delta$, i.e., $\Gamma_{1}=\left\{t_{j}^{f} \mid 0 \leq\right.$ $\left.t_{j}^{f} \leq t_{i}^{F}-\Delta\right\}$. Since $\epsilon_{i j}(s)=1$ for $s>\Delta$ [cf. (3)], we 
have $\epsilon_{i j}\left(t_{i}^{F}-t_{j}^{f}\right)=\epsilon_{i j}\left(t_{i}^{F+1}-t_{j}^{f}\right)=1$ for all $t_{j}^{f} \in \Gamma_{1}$. Thus, the sum in (12) taken over firings $t_{j}^{f} \in \Gamma_{1}$ vanishes. It follows that the sum on the right-hand side of (12) can be restricted to firings $\Gamma_{2}=\left\{t_{j}^{f} \mid t_{j}^{f}>t_{i}^{F}-\Delta\right\}$. Since $P_{\max }$ is the maximal interval that can occur in group $\Gamma_{2}$, each neuron must fire at least once in the interval $\left[t_{i}^{F}-\Delta, t_{i}^{F+1}-\Delta\right]$. We repeat the same arguments as in (14) and find $\Delta \epsilon_{i j} \geq 1$ for each unit $j$. The sum over $j$ in (16) yields

$$
P_{\max }\left(t^{*}\right) \leq 1-A .
$$

Thus, if $P_{\max }(t)$ increases once, it must either increase further or $P_{\max }(t)$ is bounded from above by $1-A$.

Step 4.-We show that an increasing $P_{\max }(t)$ is not possible. Since intervals cannot be longer than $P_{0}$, an increasing $P_{\max }(t)$ must converge to a limit $P_{1} \leq P_{0}$. On the attractor, at least one unit must assume the maximum interval $P_{1}$, and this value is approached from below. In this case, we can use the arguments of step 3 in order to show that $P_{1} \leq 1-A$. On the other hand, $1-A$ is the minimum interval. Therefore $P_{1}$ cannot be approached from below. It follows that $P_{\max }(t)$ cannot increase, and this proves part (i) of the theorem. More generally, the preceding arguments show that on every periodic solution $P_{\max }(t)=1-A$. This proves assertion (ii) of the theorem.

Step 5.-We prove part (iii) of the theorem. Let us assume that $P_{\max }(t)$ decreases at $t=t_{0}$ and stays constant during a time $t_{0} \leq t \leq t_{0}+\Delta$. This is possible only if at some point $t^{\prime}$ with $t_{0} \leq t^{\prime} \leq t_{0}+\Delta$, there is a unit $i$ with firings $t_{i}^{F}, t_{i}^{F+1}$ in the search window $\left[t^{\prime}-2 P_{0}, t^{\prime}\right]$ such that $t_{i}^{F+1}-t_{i}^{F}=P_{\max }\left(t^{\prime}\right)$ and $t_{i}^{F} \geq$ $t^{\prime}-2 P_{0}+\Delta$. Thus, $t_{i}^{F}$ is not at the beginning, but in the interior of the search interval. In this case, we can apply the arguments of steps 3 and 4 in order to derive the bound $P_{\max }\left(t^{\prime}\right) \leq 1-A$. Thus a periodic solution with $P_{\max }(t) \equiv P_{\min }$ has been reached as soon as $P_{\max }(t)$ stays constant for a time $\Delta$.

Finally, we prove the first part of the Corollary. In the situation of example (II), each unit receives connections from $n^{(1)}+n^{(2)}$ other units. Between two firings of an oscillator $i, k^{(1)} \leq n^{(1)}$ signals of strength $J^{(1)}$ and $k^{(2)} \leq n^{(2)}$ signals of strength $J^{(2)}$ may arrive. In this case, the interval of unit $i$ is $P_{0}-k^{(1)} J^{(1)}-k^{(2)} J^{(2)}$. More generally, the interval takes one out of at most $\left(n^{(1)}+1\right)\left(n^{(2)}+1\right)$ discrete values. Since $P_{\max }(t)$ must decrease at least once during a time $\Delta$, a periodic solution must be reached before $2 P_{0}+\left(n^{(1)}+1\right)\left(n^{(2)}+1\right) \cdot \Delta$. This finishes the proof.

To summarize, we have shown that a broad class of pulse-coupled oscillator networks with excitatory couplings converge to periodic solutions where every unit fires with a period $1-A$. This holds for arbitrary distributions of delays as long as the maximum delay is shorter than $1-A$. For a delay distribution with finite support, as in example (I), a periodic solution is, in general, only reached asymptotically. If there is a finite number of discrete delays, as in example (II), then the attractor is reached after a finite time. Thus phase locking is completed rapidly.

Rapid phase locking could be an important mechanism for computation in neuronal systems $[3,9,11]$. We emphasize that, on the attractor, units are phase locked but do not necessarily fire in unison and that many different solutions are possible, similar to the situation without delays [3-5]. So far our results are restricted to systems of identical oscillators without leakage terms, but we expect that our analysis can be used as a starting point for situations with a richer structure.

It is a great pleasure to thank Andreas Herz for many useful discussions and his generous hospitality. The stay at Oxford was supported by the EU under Grant No. ERB CHRX-CT92-0063.

*Present and permanent address.

[1] R. E. Mirollo and S. H. Strogatz, SIAM J. Appl. Math. 50, 1645 (1990).

[2] L.F. Abbott, J. Phys. A 23, 3835 (1990); Y. Kuramoto, Physica (Amsterdam) 50D, 15 (1991); W. Gerstner and J. L. van Hemmen, Network 3, 139 (1992); L. F. Abbott and C. van Vreeswijk, Phys. Rev. E 48, 1483 (1993); W. Gerstner and J. L. van Hemmen, Phys. Rev. Lett. 71, 312 (1993); M. Tsodyks, I. Mitkov, and H. Sompolinsky, Phys. Rev. Lett. 71, 1281 (1993); A. Treves, Network 4, 259 (1993); C. van Vreeswijk and L. F. Abbott, SIAM J. Appl. Math. 53, 253 (1993); S. Bottani, Phys. Rev. Lett. 74, 4189 (1995); U. Ernst, K. Pawelzik, and T. Geisel, Phys. Rev. Lett. 74, 1570 (1995); W. Gerstner, Phys. Rev. E 51, 738 (1995).

[3] J. J. Hopfield and A. V. M. Herz, Proc. Natl. Acad. Sci. U.S.A. 92, 6655 (1995); A. V. M. Herz and J. J. Hopfield, Phys. Rev. Lett. 75, 1222 (1995).

[4] A. Gabrielov, Physica (Amsterdam) 195A, 253 (1993); A. Gabrielov, W.I. Newman, and L. Knopoff, Phys. Rev. E 50, 188 (1994).

[5] J. E. S. Socolar, G. Grinstein, and C. Jayaprakash, Phys. Rev. E 47, 2366 (1993); A. A. Middleton and C. Tang, Phys. Rev. Lett. 74, 742 (1995).

[6] H. J.S. Feder and J. Feder, Phys. Rev. Lett. 66, 2669 (1991); Z. Olami, H. J. S. Feder, and K. Christensen, Phys. Rev. Lett. 68, 1244 (1992); A. Corral, C. P. Perez, A. Diaz-Guilera, and A. Arenas, Phys. Rev. Lett. 74, 118 (1995); M. Usher, M. Stemmler, and Z. Olami, Phys. Rev. Lett. 74, 326 (1995).

[7] J. Buck and E. Buck, Sci. Am. 234, 74 (1976).

[8] A.T. Winfree, The Geometry of Biological Time (Springer-Verlag, Berlin, Heidelberg, New York, 1980).

[9] C. M. Gray and W. Singer, Proc. Natl. Acad. Sci. U.S.A. 86, 1698 (1989); C. M. Gray, P. König, A. K. Engel, and W. Singer, Nature (London) 338, 334 (1989); R. Eckhorn et al., Biol. Cybern. 60, 121 (1988).

[10] B. Gutenberg and C.F. Richter, Ann. Geophys. 9, 1 (1956); R. Burridge and L. Knopoff, Bull. Seismol. Soc. Am. 57, 341 (1967).

[11] J. J. Hopfield, Nature (London) 376, 33 (1995). 